\title{
Controle da hemoptise maciça com broncoscopia rígida e soro fisiológico gelado*
}

\author{
Management of massive hemoptysis with the rigid \\ bronchoscope and cold saline lavage
}

\author{
Giovanni Antonio Marsico ${ }^{1}$ (Te SBPt), Carlos Alberto Guimarães ${ }^{2}$ (TE SBCT),

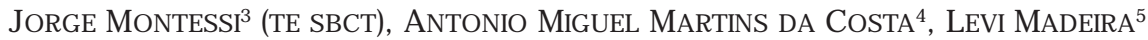

\begin{abstract}
Introdução: A hemoptise volumosa é uma condição com alta morbidade e mortalidade, independentemente do tratamento instituído. Vários métodos são utilizados para o controle do sangramento agudo. A instilação de soro fisiológico gelado através de broncoscópio rígido foi descrita em 1980.
\end{abstract}

O bjetivo: Determinar a eficácia de instilações repetidas de soro fisiológico gelado a $4^{\circ} \mathrm{C}$ através de broncoscópio rígido no controle agudo de hemoptise maciça.

Método: U ma série de 94 pacientes, com hemoptise maciça, foi tratada durante sangramento ativo com broncoscopia rígida e lavagem do pulmão sangrante com soro fisiológico gelado. Foi considerado sucesso terapêutico a ausência de sangramento nos 15 dias subseqüentes. Causas de hemoptise, com o respectivo número de pacientes: tuberculose pulmonar, $78(83 \%)$, sendo tuberculose ativa em 48 e seqüela de tuberculose em 30; bronquiectasias, seis; câncer de pulmão, cinco; aspergiloma intracavitário, três, e desconhecida, dois. 0 sítio de sangramento foi localizado em 93 pacientes (99\%). 0 volume médio de soro infundido durante a broncoscopia foi de $528 \mathrm{ml}$ e variou de $160 \mathrm{ml}$ a $2.500 \mathrm{ml}$.

Resultados: 0 sangramento cessou durante 0 procedimento em todos os pacientes. Em 15 pacientes foi feita alguma intervenção (cirurgia, embolização ou radioterapia) num prazo menor que 15 dias, e nestes a eficácia da lavagem não pôde ser avaliada. A hemoptise recorreu em 20 dos 79 pacientes acompanhados por mais de 15 dias. Houve necessidade de nova lavagem com soro fisiológico uma vez em 13 pacientes, duas vezes em seis e três vezes em um paciente.

Conclusão: 0 controle imediato da hemorragia traqueobrônquica com a administração de soro fisiológico gelado através de broncoscopia rígida é efetivo e pode ser repetido em caso de recorrência do sangramento. 0 procedimento é seguro e permite que 0 tratamento definitivo possa ser realizado em melhores condições clínicas. (J Pneumol 2003; 29(5):280-6)

Descritores - Hemoptise. Tuberculose. Broncoscopia.

* Trabalho realizado em homenagem ao Instituto de Tisiologia e Pneumologia da Universidade Federal do Rio de J aneiro, fechado em fevereiro de 2000.

1. Cirurgião de Tórax do Instituto de Doenças do Tórax e do Hosp. Municipal do Andaraí-RJ. Título de especialista pela Sociedade Brasileira de Pneumologia e Tisiologia.

2. Professor Adjunto. Cirurgião de Tórax do Instituto de Doenças do Tórax e do Hosp. Municipal do Andaraí-RJ. Título de especialista pela Sociedade Brasileira de Cirurgia Torácica.

3. Cirurgião de Tórax do Instituto de Doenças do Tórax. Professor A djunto da Universidade Federal de J uiz de Fora. Coordenador do Serviço de Cirurgia Torácica do Hospital Universitário da Universi-
Background: Massive hemoptysis is a high morbidity and high mortality condition, independently of the treatment administered. A variety of methods are used to control the acute bleeding. The instillation of iced saline solution through a rigid bronchoscope was described in 1980.

O bjective: To establish the efficacy of repeated instillations of iced saline solution $\left(4^{\circ} \mathrm{C}\right)$ using a rigid bronchoscope for the acute control of massive hemoptysis.

Method: A group of 94 patients with massive hemoptysis was treated with rigid bronchoscopy and lavage with iced saline solution of the actively bleeding lung. The absence of bleeding recurrence within the following 15 days was considered a therapeutic success. The causes of hemoptysis included: pulmonary tuberculosis: 78 (83\%), among which 48 had active disease, and 30 had tuberculosis sequelae, bronchiectasis (6), lung cancer (5), intracavitary aspergilloma (3), and unknown (2). The bleeding site was found in 93 patients $(99 \%)$. The mean saline volume used in the bronchoscopy was $528 \mathrm{~mL}$, ranging from $160 \mathrm{~mL}$ to $2,500 \mathrm{~mL}$.

Results: All patients stopped bleeding during the procedure. Fifteen patients were submitted to some kind of procedure (surgery, embolization, or radiation therapy) within 15 days, and the efficacy of lavage could not be assessed. Twenty of the 79 patients followed-up for more than 15 days had recurrence of hemoptysis. Cold saline lavage was repeated once in 13 patients, twice in 6 patients, and 3 times in one patient.

Conclusion: The control of tracheobronchial hemorrhage through bronchoscopy and lavage with iced saline is an effective procedure and can be repeated in case of re-bleeding. It is a safe procedure, and allows the definitive treatment to occur in better clinical conditions.

Key words - Hemoptysis. Tuberculosis. Bronchoscopy.

dade Federal de J uiz de Fora. Título de especialista pela Sociedade Brasileira de Cirurgia Torácica.

4. Pneumologista do Instituto de Doenças do Tórax.

5. Professor A ssistente da Faculdade de Medicina Gama Filho - Rio de J aneiro. Ex-Cirurgião de Tórax do Instituto de Tisiologia e Pneumologia.

Endereço para correspondência - Giovanni Antonio Marsico, Hospital Municipal do Andaraí, RJ - Cirurgia Torácica, 70 andar, Rua Leopoldo, 280 - 21541-170 - Rio de J aneiro, RJ. Tel.: (21) 2575-7035; email: marsicog@gbl.com

Recebido para publicação em 2/12/02. Aprovado, após revisão, em 14/6/03. 


\section{INTRODUÇÃO}

A hemoptise volumosa, independente do tratamento instituído, apresenta altas taxas de morbidade e mortalidade. Os critérios utilizados para caracterizar a hemoptise como maciça são variáveis, baseados fundamentalmente no volume de sangue eliminado em um determinado período, que varia de $100 \mathrm{ml}$ a $600 \mathrm{ml}$ ou mais, em 24 horas. $^{(1-5)}$

Quase sempre a morte ocorre por sufocação e raramente por anemia aguda. Dos doentes não operados, embora tivessem indicação e condições clínicas para tal, $80 \%$ morrem ao término de um ano em virtude de nova hemoptise. Nas hemoptises volumosas o risco de morte por asfixia é iminente, os doentes exigem cuidados intensivos, vigilância constante e não devem ser mantidos e segregados em locais isolados. São múltiplos os relatos de morte súbita provocada por novo episódio de sangramento, enquanto os pacientes aguardavam esclarecimento diagnóstico e tratamento. ${ }^{(2,4,6,7)}$

Diversos são os fatores que influenciam na evolução dos pacientes com hemoptise volumosa e controversa a caracterização da hemorragia pulmonar. 0 volume de sangue eliminado é importante, mas não o único fator determinante. Devemos considerar que: 0 volume da hemoptise exteriorizada nem sempre corresponde ao total da hemorragia, pois boa parte do sangue pode estar retido na árvore traqueobrônquica; episódio único de hemoptise maciça envolve risco maior de obstrução das vias aéreas e inundação alveolar, do que aqueles em que há sangramento da mesma quantidade em vários episódios durante o período de $24 \mathrm{~h}$; hemoptises pequenas e persistentes são capazes de gradativamente deteriorar a condição clínica e a função pulmonar; a tosse é um mecanismo de defesa eficaz contra a asfixia, entretanto freqüentemente está ausente em doentes debilitados; em pacientes com a função pulmonar previamente comprometida, de forma temporária ou definitiva, a quantidade de sangue capaz de causar asfixia é menor; o mecanismo de sangramento como o causado por fístula entre a aorta e o pulmão, ou o originado de abscesso pulmonar, são considerados de controle mais difícil do que o proveniente de um infiltrado pulmonar; doenças graves associadas como cardiopatias, insuficiência renal, diabetes e outras, pioram o prognóstico; podem existir coagulopatias ou uso de anticoagulantes. Portanto, com base nessas considerações, foi sugerido o abandono do vocábulo "maciça" e a adoção da definição mais abrangente "hemoptise que ameaça a vida". ${ }^{(1,2,4,5,8,9-13)}$

Vários são os métodos utilizados para o controle da hemoptise que ameaça a vida, tais como: embolização das artérias brônquicas, lavagem endobrônquica com soro fisiológico gelado, pneumoperitônio, pneumotórax, blo- queios brônquicos, intubação brônquica seletiva, uso do laser, da vasopressina e outros. Conlan e Hurwitz, ${ }^{(14)} \mathrm{em}$ 1980 , mostraram a possibilidade de controlar a hemoptise maciça com broncoscopia rígida e lavagem endobrônquica regrada com soro fisiológico gelado a $4^{\circ} \mathrm{C}$.

0 objetivo do presente trabalho foi verificar a eficácia aguda e tardia da lavagem endobrônquica com soro fisiológico gelado a $4^{\circ} \mathrm{C}$ através de broncoscopia rígida no controle da hemoptise maciça.

\section{MÉTOdo}

No Instituto de Tisiologia e Pneumologia da Universidade Federal do Rio de J aneiro, entre julho de 1984 e julho de 1990, foram estudados prospectivamente 94 pacientes que ap resentaram hemoptise de $400 \mathrm{ml}$ ou mais em 24 horas. Os doentes foram submetidos a broncoscopia rígida e lavagem endobrônquica com soro fisiológico gelado a $4^{\circ} \mathrm{C}$, em vigência de sangramento. A idade variou de 17 a 72 anos (média de 34 anos), sendo que 65 eram do sexo masculino.

0 atendimento foi padronizado em todos os pacientes: radiografia de tórax; punção de veia profunda, com preferência pela veia jugular interna direita; reposição de sangue (somente com hematócrito abaixo de 30\%); dosagem dos gases arteriais, monitorização cardíaca e oximetria; quando possível, foi utilizado pré-anestésico: $1 \mathrm{mg} /$ $\mathrm{kg}$ de meperidina, associada a $0,5 \mathrm{mg}$ de atropina intramuscular; anestesia tópica com xilocaína a $2 \%$, pela via transglótica ou transcricotireoidiana; para sedação, no início do estudo foi utilizado benzodiazepínico intravenoso e, posteriormente, midazolam intravenoso; broncoscopia rígida.

Sob oxigênio contínuo, com os pacientes em decúbito dorsal e coxim transversal posicionado sob os ombros, o broncoscópio rígido foi introduzido nos 94 pacientes que sangravam ativamente no momento do exame. Após ultrapassar as cordas vocais e alcançar a traquéia, por meio de aspirações sucessivas, procurávamos retirar o máximo possível de sangue, de forma que nos permitisse identificar pelo menos o brônquio principal de onde provinha 0 sangramento. Em seguida, o brônquio sangrante era intubado seletivamente. Iniciávamos a lavagem endobrônquica com soro fisiológico a $4^{\circ} \mathrm{C}$, instilando por vez o volume de $30 \mathrm{ml}$ a $50 \mathrm{ml}$, aspirados a intervalos sucessivos de 10 a 20 segundos. As lavagens eram repetidas até cessar o sangramento. Durante o procedimento, procurávamos identificar o brônquio lobar ou segmentar responsável pela drenagem do sangue. Com freqüência o broncoscópio era recuado até a traquéia para permitir melhor ventilação do pulmão não sangrante. 0 broncoaspirado colhido era enviado para pesquisa de células neoplásicas, pesquisa direta e cultura para fungos, pes- 
quisa direta de bacilo álcool-acidorresistente e cultura para micobactérias. Outros exames eram solicitados de acordo com as suspeitas diagnósticas.

A pós o controle inicial da hemoptise, os pacientes eram submetidos às seguintes medidas: internação em unidade de cuidados intensivos; posicionamento no leito com a cabeceira baixa, pés elevados e decúbito lateral sobre o lado do sangramento; manutenção sob oxigênio contínuo; feitas radiografias de tórax e dosagens diárias dos gases arteriais; administração de derivados da morfina para diminuir o reflexo da tosse e leve sedação; uso de antibióticos com amplo espectro de ação; realização de broncofibroscopia flexível nos dias subseqüentes para limpeza da árvore brônquica; na suspeita clínica e radiológica de tuberculose pulmonar em atividade, iniciávamos imediatamente o tratamento com drogas antituberculose enquanto aguardávamos a confirmação bacteriológica.

Os pacientes foram acompanhados por no mínimo seis meses. Consideramos como controle eficaz da hemoptise maciça, com broncoscopia rígida e lavagem endobrônquica com soro fisiológico gelado a $4^{\circ} \mathrm{C}$, o tempo mínimo de 15 dias com o paciente sem sangrar. Durante esse período é possível o tratamento clínico e que as drogas exerçam os seus efeitos e, nos pacientes passíveis de tratamento cirúrgico, o estudo pré-operatório e o preparo adequado.

\section{REsultados}

A tuberculose pulmonar, nas suas várias formas, incidiu em 78 (83\%) pacientes: 41 (43,6\%) com tuberculose ativa sensível às drogas, sete $(7,4 \%)$ com tuberculose multirresistente e 30 (32\%) com seqüelas de tuberculose.

Bronquiectasias foram diagnosticadas em seis $(6,4 \%)$ pacientes, câncer de pulmão em cinco $(5,3 \%)$, micetoma intracavitário em três (3,2\%), e em dois pacientes não foi possível estabelecer o diagnóstico: em um o estudo não foi considerado adequado e, no outro, o estudo anatomopatológico da biópsia pulmonar não foi elucidativo (Tabela 1).

\section{TABELA 1}

Causas de hemoptise maciça em 94 pacientes

\begin{tabular}{lrc}
\hline \multicolumn{1}{c}{ Diagnóstico } & N & $\%$ \\
\hline Tuberculose ativa & 41 & $(43,6)$ \\
Tuberculose residual & 30 & $(32)$ \\
Tuberculose multidrogarresistente & 7 & $(7,4)$ \\
Bronquiectasia & 6 & $(6,4)$ \\
Câncer de pulmão & 5 & $(5,3)$ \\
Micetoma intracavitário & 3 & $(3,2)$ \\
Ignorado & 2 & $(2,1)$ \\
Total & 94 & $(100)$ \\
\hline
\end{tabular}

Relato de hemoptise prévia, variando desde hemoptóicos até grandes volumes, ocorreu em $40(42,5 \%)$ dos 94 doentes. A incidência foi maior nas doenças inflamatórias crônicas e nas seqüelas da tuberculose: em 56,7\% desses pacientes.

O controle imediato da hemorragia pulmonar, com broncoscopia rígida e uso de soro fisiológico gelado a $4^{\circ} \mathrm{C}$, foi obtido nos 94 pacientes. 0 volume de soro fisiológico gelado a $4{ }^{\circ} \mathrm{C}$ utilizado nas lavagens variou de $160 \mathrm{ml} \mathrm{a}$ $2.500 \mathrm{ml}$ (média $582 \mathrm{ml}$ ). 0 tempo médio de duração do procedimento foi de 30 minutos.

A pós o procedimento, sempre que possível foram acrescidos outros métodos de controle ou então o tratamento definitivo: clínico, cirúrgico ou radioterápico. Em 15 pacientes não foi possível avaliar a eficácia da lavagem endobrônquica no prazo de 15 dias. Durante esse intervalo, com a hemoptise controlada, os doentes foram submetidos ao tratamento cirúrgico, embolização das artérias brônquicas ou radioterapia.

Em virtude de novo episódio de hemoptise, a repetição da lavagem endobrônquica com soro fisiológico gelado se fez necessária, no espaço de 15 dias, em 20 (25,3\%) dos 79 pacientes. Em 13 foi repetida uma vez, em seis duas vezes e em um paciente três vezes. 0 maior percentual de repetição ocorreu naqueles com tuberculose pulmonar ativa, ou seja, em 11 (26,8\%) dos 41 pacientes. A nova lavagem com soro fisiológico gelado foi necessária no período que variou de 12 horas até nove dias, sendo que em seis foi repetida nos dois primeiros dias.

Foram realizadas 122 broncoscopias rígidas, todas em vigência de sangramento. Na primeira broncoscopia o pulmão sangrante foi identificado em 93 (99\%) dos 94 pacientes. Em 54 (57,5\%), com origem no pulmão direito e $36(38,7 \%)$ no esquerdo. Sangramento bilateral, alternado nos lobos superiores, foi verificado em três $(3,2 \%)$ pacientes. A localização lobar do sangramento foi possível em 49 (52,7\%) ocasiões: nos lobos superiores em 29 , nos inferiores em 19 e no lobo médio em uma ocasião. A preocupação da localização lobar nem sempre esteve presente, principalmente, nos pulmões destruídos pela doença, já que apresentavam estenose brônquica parcial e grande distorção da árvore brônquica. Essa condição, com alguma freqüência, não permitia a introdução do broncoscópio rígido e das óticas além da entrada do brônquio principal.

Nos 30 doentes com seqüelas pulmonares da tuberculose, $18(60 \%)$ apresentavam comprometimento bilateral enquanto que nos pacientes com tuberculose multirresistente, quatro $(57 \%)$ dos sete pacientes 0 apresentavam. Dentre os 61 (65\%) pacientes com lesão pulmonar unilateral, nove $(14,7 \%)$ morreram. No grupo de 33 (35\%) com lesões bilaterais, 15 (45,4\%) faleceram. O correu grande inundação alveolar por sangue, uni ou bilateral, em 14 
(15\%) doentes. Destes, 10 eram portadores de tuberculose pulmonar ativa, sendo que sete com lesões unilaterais morreram e três com doença bilateral sobreviveram. Dos quatro doentes restantes, com seqüelas pulmonares bilaterais da tuberculose, três morreram. No total morreram $10(71,4 \%)$ dos 14 pacientes com grande inundação alveolar.

Dentre os 41 pacientes com tuberculose pulmonar ativa sensíveis às drogas, 23 eram virgens de tratamento $e$ $18 \mathrm{com}$ tuberculose pulmonar recorrente. A presentavam lesões unilaterais $33(80,5 \%)$ pacientes e bilaterais oito $(19,5 \%)$ deles. Em $30(73,2 \%)$ doentes, inicialmente a hemoptise foi controlada com lavagem endobrônquica e soro fisiológico gelado e foram então tratados com rifampicina, hidrazida e pirazinamida: $25(83,3 \%)$ apresentaram cura e cinco $(16,7 \%)$ morreram. Somente um óbito foi causado por nova hemoptise e sepse, nove dias após o primeiro episódio. As outras causas foram: insuficiência respiratória: um; insuficiência hepática e desnutrição: um; e sepse: dois óbitos. Desde a chegada ao hospital nenhum deles apresentava condição de ser submetido ao tratamento cirúrgico. Após o controle inicial com soro fisiológico gelado, em sete $(17,1 \%)$ pacientes foram realizadas embolizações arteriais brônquicas. 0 único óbito foi devido a novo episódio de hemoptise 24 horas depois da embolização e nove dias após o primeiro episódio de sangramento. Q uatro pacientes com tuberculose pulmonar sensíveis às drogas foram submetidos a ressecção pulmonar, um porque recusou a medicação específica, um por apresentar hemoptise maciça após três meses de tratamento correto e culturas negativas, um sem diagnóstico prévio e um que evoluiu com coágulo encravado na entrada do brônquio do lobo superior direito, o qual não foi possível retirar mesmo após o controle do sangramento. A ressecção pulmonar foi indicada pelo risco do coágulo desprender-se e causar nova hemorragia. $\mathrm{Na}$ análise total dos 41 pacientes com tuberculose pulmonar ativa

TABELA 2

Controle da hemoptise maciça com broncoscopia e soro gelado em 41 pacientes com tuberculose pulmonar ativa - sensíveis as drogas

\begin{tabular}{lrrrrr}
\hline \multicolumn{1}{c}{ Tratamento } & Pacientes \% & ó bitos \% & $\begin{array}{c}\text { Cura seis } \\
\text { meses (\%) }\end{array}$ \\
\hline BCP, SG, RHZ & 30 & $(73,2)$ & $* 5(16,7)$ & 25 & $(83,3)$ \\
BCP, SG, RHZ, EAB & 7 & $(17,1)$ & $* 1(14,3)$ & 6 & $(85,7)$ \\
BCP, SG, RHZ, CIR & 3 & $(7,3)$ & $2(66,7)$ & 1 & $(33,3)$ \\
BCP, SG, RHZ, EAB, CIR & 1 & $(2,4)$ & - & $1(100)$ \\
Total & $41(100)$ & $8(19,5)$ & 33 & $(80,5)$ \\
\hline
\end{tabular}

BCP - broncoscopia; SG - soro gelado; RHZ - rifampicina, hidrazida, pirazinamida; EAB embolização artéria brônquica; CIR - cirurgia.

* má função pulmonar e mau estado clínico. sensíveis às drogas, que foram submetidos a vários métodos de controle e tratamento da hemoptise maciça, oito $(19,5 \%)$ morreram e $33(80,5 \%)$ apresentaram cura (Tabela 2).

Dos 30 pacientes com tuberculose pulmonar residual, $18(60 \%)$ eram portadores de lesões pulmonares bilaterais e $12(40 \%)$ unilaterais. Para controle da hemoptise, em 12 pacientes utilizamos apenas broncoscopia rígida e soro fisiológico gelado a $4{ }^{\circ} \mathrm{C}$, todos com contra-indicação para tratamento cirúrgico, principalmente, por má função pulmonar. Neste grupo, cinco $(41,7 \%)$ morreram nos primeiros seis meses de acompanhamento, com nova hemoptise maciça. Em cinco (16,7\%) pacientes sem condições clínicas para tratamento cirúrgico, foi acrescida embolização arterial brônquica, e três $(60 \%)$ faleceram, um com insuficiência respiratória e sepse e dois com nova hemoptise maciça. Ao fim de seis meses de acompanhamento dois (40\%) estavam vivos. Em dois doentes com a hemoptise controlada após lavagem endobrônquica com soro fisiológico gelado e embolização das artérias brônquicas foi realizada ressecção pulmonar. A mbos morreram por complicações no pós-operatório. No grupo de $11(36,6 \%)$ pacientes que foram operados eletivamente, após terem sido submetidos a lavagem endobrônquica com soro fisiológico gelado, ocorreu um $(9,1 \%)$ óbito (Tabela 3).

A companhamos sete pacientes com tuberculose multidrogarresistente. Inicialmente, a hemoptise foi controlada com soro gelado. Entre seis e 10 dias, três morreram com insuficiência respiratória e sepse, todos sem condições clínicas de serem submetidos ao tratamento cirúrgico. Três foram operados, dois foram curados e um faleceu. Em um paciente, também foi realizada embolização arterial brônquica, pois este recusou o tratamento cirúrgico. Foi acompanhado por seis meses sem recorrência da hemoptise.

Dos seis pacientes com bronquiectasias, após controle inicial da hemoptise com soro gelado e preparo pré-ope-

TABELA 3

Controle da hemoptise maciça com broncoscopia e soro gelado em $\mathbf{3 0}$ pacientes com tuberculose pulmonar residual

\begin{tabular}{lrrrrc}
\hline $\begin{array}{c}\text { Controle } \\
\text { tratamento }\end{array}$ & Pacientes \% & ó bitos \% & $\begin{array}{c}\text { Sem hemoptise } \\
\text { seis meses } \%\end{array}$ \\
\hline BCP e SG & $* 12$ & $(40)$ & $* * 5$ & $(41,7)$ & $7(58,3)$ \\
BCP, SG, EAB & $* 5$ & $(16,7)$ & $* * 3(60)$ & $2(40)$ \\
BCP, SG, EAB, CIR & 2 & $(6,7)$ & $2(100)$ & - \\
BCP, SG, CIR & 11 & $(36,6)$ & 1 & $(9)$ & $10(91)$ \\
Total & $30(100)$ & $11(36,7)$ & $19(63,3)$ \\
\hline
\end{tabular}

BCP - broncoscopia; SG - soro gelado; CIR - cirurgia; EAB - embolização artéria brônquica * com contra-indicação cirúrgica

** 6 morreram com nova hemoptise e 2 por complicações da hemoptise 
ratório, cinco foram operados e curados. Um recusou a cirurgia e seis meses após mantinha-se sem sangrar.

Os cinco pacientes com câncer de pulmão possuíam tumores pulmonares grandes, irresecáveis e centrais. A pós o controle da hemoptise com soro fisiológico gelado, três foram submetidos a radioterapia e não repetiram o sangramento em seis meses, e dois morreram sem hemoptise antes de seis meses.

A pós o controle inicial com soro fisiológico gelado, os três doentes com micetoma pulmonar intracavitário foram submetidos ao tratamento cirúrgico com boa evolução.

Os dois doentes cuja causa da hemoptise não foi esclarecida evoluíram durante um ano sem recidiva de sangramento.

\section{DISCUSSÃO}

A hemoptise volumosa possui caráter recidivante e evolui com morbidade e mortalidade altas. A o estabelecer-se a seqüência controle da hemoptise, diagnóstico e tratamento, os resultados melhoram. . $2,6,15-18)^{-1}$

A tuberculose pulmonar nas diferentes formas é a principal causa de hemoptise maciça, com incidência de $52 \%$ a $73 \%$. A tuberculose pulmonar ativa contribui com 38\% a $50 \%$ dos casos. Em países desenvolvidos as bronquiectasias ocupam o primeiro lugar. ${ }^{(4,15-20)} \mathrm{C}$ onlan e H urwitz ${ }^{(14)}$ publicaram em 1980 os resultados obtidos em 12 pacientes que tiveram hemoptise maciça, $600 \mathrm{ml}$ ou mais em $24 \mathrm{~h}$, controlada com broncoscopia rígida sob anestesia geral e lavagem endobrônquica com soro fisiológico gelado a $4{ }^{\circ} \mathrm{C}$. Posteriormente, Conlan et al., ${ }^{(15)} \mathrm{em} 1983$, agora em 23 doentes, confirmaram os resultados. 0 volume utilizado de soro fisiológico gelado variou de $300 \mathrm{ml}$ a $750 \mathrm{ml}$, com média de $500 \mathrm{ml}$. A hemoptise provavelmente cessa em virtude da vasoconstricção e do espasmo arterial induzido pela ação do soro gelado.

A redução do fluxo sanguíneo propicia a formação de trombos e a oclusão dos vasos. ${ }^{(2,14,15)}$ O btivemos o controle imediato da hemoptise em nossos 94 pacientes. 0 volume de soro fisiológico gelado utilizado variou de $160 \mathrm{ml}$ a $2.500 \mathrm{ml}$, com média de $582 \mathrm{ml}$. Sob anestesia tópica e sedação, realizamos 122 broncoscopias rígidas e lavagem endobrônquica com soro gelado a $4^{\circ} \mathrm{C}$. O procedimento permitiu controlar o sangramento, definir o sítio da hemoptise e o diagnóstico. A broncoscopia não acarretou nenhum malefício no sentido de exacerbar o sangramento. A broncoscopia sob narcose implica em algumas desvantagens: risco do ato anestésico, a necessidade de experiência do anestesiologista e a sala de operações disponível a qualquer momento.(1)

Nas grandes hemoptises é imperativo o uso do broncoscópio rígido. 0 amplo conduto permite ventilação pulmonar adequada, rápida e eficiente aspiração de sangue e coágulos, boa visão endoscópica e intubação seletiva do pulmão. Os principais óbices ao uso do broncofibroscópio são: canal de aspiração pequeno que obstrui facilmente com sangue e coágulos, observação endoscópica difícil, pois freqüentemente a lente é obscurecida pelo sangue, e ventilação pulmonar inadequada. A pós controlar o sangramento o aparelho flexível pode ser introduzido pela luz do broncoscópio, tubo orotraqueal e cânula de traqueostomia. ${ }^{(7,9,15,16,18,21-23)}$

Identificando-se previamente o sítio de sangramento é possível a execução de embolizações e cirurgias precisas e seletivas. Somente o exame endoscópico localiza com certeza o sangramento. ${ }^{(24-31)}$ Garzon et al.,(21) com broncoscopia rígida, identificaram o sítio da hemoptise em 74 (100\%) pacientes. A literatura mostra $85 \%$ a $100 \%$ de acertos. $(2,6,9,16,17,31,32)$

Identificamos o sítio da hemoptise em 93 (99\%) dos 94 pacientes, e ele quase sempre correspondia à lesão radiológica pulmonar única ou à mais exuberante. Verificamos que ocorreu hemoptise, em momentos diferentes, em ambos os lobos superiores em três $(3,2 \%)$ dos nossos 33 pacientes com lesões bilaterais. Portanto, é conveniente repetir a broncoscopia a cada novo episódio de sangramento.

Com alguma freqüência, encontramos verdadeiros moldes da árvore traqueobrônquica formados por coágulos que causaram atelectasia. A retirada endoscópica acarreta sangramento intenso, ocasionado pela liberação do sangue que se encontrava retido. A remoção é necessária, mas com o broncofibroscópio é extremamente perigosa. Algumas das mortes súbitas, caracterizadas como "hemoptises fulminantes", provavelmente, ocorrem após o desprendimento repentino de coágulos e a liberação do sangue represado. A broncofibroscopia, realizada nos dias subseqüentes ao da hemorragia, foi preponderante para a toalete traqueobrônquica.

A proximadamente $80 \%$ das hemoptises volumosas originam-se nas artérias brônquicas, $7 \%$ nas artérias pulmonares e 7\% no sistema arterial não-brônquico.(24-26,32-36) Remy et al.,(24-26) com a embolização das artérias brônquicas, obtiveram o controle imediato da hemoptise maciça em $85 \%$ dos pacientes.

Nos primeiros 15 dias, após a lavagem endobrônquica com soro fisiológico gelado, em 79 pacientes não acrescentamos outro método de controle ou tratamento cirúrgico. Nova lavagem endobrônquica foi necessária em 20 $(25,3 \%)$ pacientes. Consideramos o tempo de 15 dias suficiente para o diagnóstico e o estabelecimento do tratamento, clínico ou cirúrgico.

Garzon e Gourin ${ }^{(18)}$ verificaram que, nas cirurgias realizadas durante hemoptise, $33 \%$ dos pacientes morreram. Com sangramento brando, 7\% faleceram. A inundação 
alveolar por sangue é uma complicação temida, com morbidade e mortalidade altas. A condição ideal no atendimento é transformar a cirurgia de emergência em eletiva, com a hemoptise controlada e a função pulmonar conhecida. Função pulmonar limítrofe reforça a indicação cirúrgica, pois o risco de morte por asfixia aumenta. ${ }^{(6,7,17,37,38)}$

O comprometimento da função pulmonar, temporária ou definitiva, é fator agravante. A companhamos 14 pacientes com grande inundação alveolar, e 10 (71,4\%) morreram. Dentre os 33 que tinham doença pulmonar bilateral, $15(45,4 \%)$ morreram. A traqueostomia é considerada medida salvadora naqueles que evoluem com inundação alveolar por sangue e/ ou nos portadores de doença pulmonar extensa. Facilita a ventilação pulmonar, a aspiração de sangue ou de secreções e funciona como preparo pré-operatório.

Com o decorrer do nosso estudo verificamos que invariavelmente todos que sangraram de forma volumosa evoluíram com infecção broncopulmonar, principalmente aqueles com lesões pulmonares cavitárias grandes.

O correram sete mortes causadas diretamente pela infecção. Portanto, passamos a prescrever antibióticos com amplo espectro de ação para germes inespecíficos. Conlan et al. ${ }^{(15)}$ controlaram e trataram com broncoscopia rígida, soro fisiológico gelado a $4^{\circ} \mathrm{C}$ e tuberculostáticos, 11 pacientes com tuberculose pulmonar ativa e hemoptise maciça, e nove $(81,8 \%)$ apresentaram cura.

Dentre os nossos 41 pacientes com tuberculose pulmonar ativa, após o controle inicial do sangramento com broncoscopia e lavagem endobrônquica com soro fisiológico gelado, em 30 deles associamos rifampicina, hidrazida e pirazinamida, e $25(83,3 \%)$ sobreviveram. Verificamos que, após 10 dias, não ocorreu recidiva do sangramento. Os cinco $(16,7 \%)$ pacientes que morreram não apresentavam condições para tratamento cirúrgico e apenas uma das mortes foi por nova hemoptise, após nove dias de uso das drogas antituberculose. Em 11 (26,8\%) pacientes foram acrescentados outros métodos de controle ou tratamentos definitivos em menos de 15 dias, com a hemoptise controlada. Garzon e Gourin ${ }^{(18)}$ operaram 33 pacientes com tuberculose pulmonar ativa, 25 em vigência de hemoptise maciça. Crocco et al.(4) nas mesmas condições operaram 16 pacientes. Os autores não utilizaram métodos de controle. A maioria das ressecções pulmonares talvez pudessem ter sido evitadas, pois controlando-se a hemoptise, os tuberculostáticos disporiam de tempo suficiente para exercer sua ação.

Dos 30 pacientes com seqüelas de tuberculose pulmonar e hemoptise maciça, somente $13(43,3 \%)$ apresentavam condições para o tratamento operatório. Em 12 (40\%) a hemoptise foi controlada somente com broncoscopia rígida e lavagem endobrônquica com soro gelado. Estes puderam então ser estudados e avaliados já com a hemorragia sustada, porém, todos se apresentaram sem função pulmonar que permitisse ressecção pulmonar. Ao final de seis meses cinco $(41,7 \%)$ morreram em virtude de nova hemoptise. Após lavagem endobrônquica com soro fisiológico gelado, associamos embolização arterial brônquica em sete $(23,3 \%)$ pacientes, dos quais, cinco $(71,4 \%)$ morreram, sendo que dois em virtude de nova hemoptise, um por complicações infecciosas, e dois operados com função pulmonar limítrofe, por complicações no pós-operatório. Dos $11(36,7 \%)$ pacientes que foram operados com a hemoptise controlada com broncoscopia e soro fisiológico gelado, 10 (91\%) sobreviveram e um $(9 \%)$ morreu.

Segundo a literatura, nos doentes com seqüelas pulmonares da tuberculose e hemoptise volumosa, a mortalidade operatória varia de $26 \%$ a $36 \%$. $4,6,15,17-19,22,32)$

Com broncoscopia rígida e soro fisiológico gelado a $4^{\circ} \mathrm{C}$, o controle imediato da hemoptise maciça foi obtido por Conlan et al. ${ }^{(15)}$ em oito pacientes com bronquiectasias, sendo que três foram operados e cinco manejados clinicamente. Da mesma maneira controlamos seis paciente com bronquiectasias, permitindo o estudo e o mapeamento adequado da árvore brônquica. Cinco foram operados e curados, e um recusou a cirurgia e durante seis meses não sangrou.

Hemoptise volumosa no câncer de pulmão é pouco freqüente, acometendo de $4 \%$ a $7,5 \%$ dos pacientes. Os tumores, geralmente, são centrais e irressecáveis. ${ }^{(35,36)}$ Conlan et al. (15) controlaram com soro fisiológico gelado seis pacientes, sendo que três foram tratados com medidas conservadoras e três submetidos a radioterapia.

A companhamos cinco $(5,3 \%)$ pacientes com câncer de pulmão e hemoptise maciça, e todos estavam em estado bastante avançado da doença. Foram controlados com soro fisiológico gelado e mantidos durante 15 dias sem sangramento. Três foram submetidos a radioterapia e dois, sem condições clínicas para qualquer tipo de tratamento, morreram sem hemoptise após três meses. A embolização arterial brônquica apresenta resultados precários a curto e a longo prazo nos casos de micetoma pulmonar intracavitário e hemoptise. ${ }^{(27,36)}$

A companhamos três doentes com colonização fúngica da caverna tuberculosa e hemoptise volumosa, a qual foi imediatamente controlada com soro fisiológico gelado. Dois dias após o procedimento, um doente foi submetido a cavernostomia e, após 15 dias, dois a ressecção pulmonar. Concluímos que: a lavagem endobrônquica com soro fisiológico gelado a $4^{\circ} \mathrm{C}$ é um método inicial de controle eficaz nos pacientes com hemoptise de $400 \mathrm{ml}$ ou mais em 24h; em vigência de hemoptise maciça, foi possível determinar o pulmão sangrante em $99 \%$ dos pacientes; nos doentes com tuberculose pulmonar ativa, o controle da 
hemoptise com broncoscopia rígida, soro fisiológico gelado a $4^{\circ} \mathrm{C}$ e drogas antituberculose evitou a ressecção pulmonar e permitiu a cura em $83,3 \%$ dos pacientes; a

\section{REFERÊNCIAS}

1. Marsico GA, Montessi J. Hemoptise. In: Tarantino AB, editor. Doenças pulmonares. 5a ed. Rio de J aneiro: Guanabara Koogan, 2002;22-32.

2. Conlan AA. Massive hemoptysis - Diagnostic and therapeutic implications. Ann Surg 1985;17:337-54.

3. Bobrowitz ID, Ramakishma S, Shim YS. Comparison of medical v. surgical treatment of major hemoptysis. Arch Intern Med 1983;143: 1246-343.

4. Crocco JA, Rooney JJ, Frankaushen OS, Di Benedetto RJ, Lyons HA. Massive hemoptysis. Arch Intern Med 1968;121:495-8.

5. A mirana M, Freter $R$, Tirschwell $P$, J anis $M$, Blomberg A, State D. No aggressive surgical approach to significant hemoptysis in patient with pulmonary tuberculosis. Am Rev Respir Dis 1968;97:187-92.

6. Marsico GA. Controle da hemoptise maciça com broncoscopia e soro gelado [Tese]. Rio de J aneiro, UFF; 1991.

7. Marsico GA. Hemoptise maciça - Métodos de controle. JBM 1994; $66: 19-36$

8. Laforet EG, Steider JW. Subcostal extraperiostal plombage for massive tuberculous hemoptysis. Am Rev Respir Dis 1960;81:397-402.

9. MCCollum WB, Mattox KL, Grinm GA, Beall AC. Immediate operative treatment for massive hemoptysis. Chest 1975;62:152-5.

10. Pursel SE, Lindiskog GE. Hemoptysis. Am Rev Respir Dis 1961;84: 329-36.

11. Teklu B, Felleke G. Massive hemoptysis in tuberculosis. Tubercle 1982; 63:213-6.

12. Haponik EF, Chin R. Hemoptysis clinician's perspectives. Chest 1990 97:469-75.

13. Guimarães CA. Massive hemoptysis. In: Pearson FG, editor. Thoracic surgery. New York: Churchill Livingstone, 2001;717-35.

14. Conlan AA, Hurwitz SS. Management of massive hemoptysis with the rigid bronchoscope and cold saline lavage. Thorax 1980;35:901-4.

15. Conlan AA, Hurwitz SS, Kigel GA, Nicolau N, Pool R. Massive hemoptysis. J Thorac Cardiovasc Surg 1983;85:120-4.

16. Guimarães CA, Marsico GA, Montessi J, Clemente AM, Saito EH, Fonseca CAS. O papel da broncoscopia na hemoptise maciça. Pulmão RJ 1999;8:256-62.

17. Guimarães $C A$. Tratamento cirúrgico da hemoptise maciça [Tese]. Rio de J aneiro, UFRJ -ITP, 1992.

18. Garzon AA, Gourin A. Surgical management of massive hemoptysis. Ann Surg 1978;187:267-71.

19. Sehat $S$, Oreizie M, Moinedine K. Massive pulmonary hemorrhage: surgical approach as choice of treatment. Ann Thorac Surg $1978 ; 25: 12-5$

20. Middleton J R, Sem Plange M, Salani J, Kapila R, Louria DB. Deathproducing hemoptysis in tuberculosis. Chest 1997;72:601-4. lavagem endobrônquica com soro fisiológico gelado a $4^{\circ} \mathrm{C}$ permitiu realizar ressecções pulmonares com a hemoptise controlada.

21. Garzon AA, Cerruti MM, Golding ME. Exsanguinant hemoptysis. J Thorac Cardiov Surg 1982;84:829-33.

22. Gourin A, Garzon AA. Operative treatment of massive hemoptysis. Ann Thorac Surg 1974;18:52-60.

23. Gourin A, Garzon AA. Control of hemorrhage in emergency pulmonary resection for massive hemoptysis. Chest 1975;68:120-5.

24. Remy J, Arnaud A, Fardou LL, Gibaud LI. Treatment of hemoptysis by embolization of bronchial arteries. Radiology 1977;122:33-7.

25. Remy J, Lemaitre L, Lafite J J, Vilmn MD, Michel J S. Massive hemoptysis of pulmonary arterial origin. AJ R 1984;143:963-9.

26. Rémy-J ardin $M$, Rémy J . La vascularisation systémique non bronchique du poumon. Rev Fr Mal Resp 1990;7:95-118.

27. Sahebjamj AB. Feed saline lavage during bronchoscopy. Chest 1975; 69:131-2

28. Yeoh CB, Hhbaytar RT, Ford JM, Wylie RH. Treatment of massive hemorrhage in pulmonary tuberculosis. J Thorac Cardiovasc Surg 1967; 54:503-10.

29. Haponik EF, Fein A, Chin R. Managing life-threatening hemoptysis: has anything really changed? Chest 2000;118:1431-5.

30. Freitag AP, Tekolf E, Stamatis G. Three year experience with a new balloon catheter for the management of hemoptysis. Eur Respir J 1994; 7:2033-7

31. Dweik RA, Stoller J K. Flexible bronchoscopy in the $21^{\text {st }}$ century - Role of bronchoscopy in massive hemoptysis. Clin Chest Med 1999;20:89105.

32. Garzon AA, Cerruti M, Gourin A, Karlson KE. Pulmonary resection for massive hemoptysis. Surgery 1970;67:633-8.

33. A belanet $R$, Davssy $M$, Deslignères $S S$. A pport de l'anatomie pathologique a la compréhension des hémoptisies. Rev Fr Mal Resp 1976;4: 693-706.

34. Tobin CE. The bronchial arteries and their connections with other vessels in the human lung. Surg Gyn O bst 1952;95:741-50.

35. U flacker R, Kaemmere A, Picon PD, Rizzon CFO, News CMC, Oliveira $E S B$, et al. Bronchial artery embolization in the management of hemoptysis: technical aspects and long-term results. Radiology 1985; 157:637-44.

36. Mal H, Rullon I, Mellot F, Brugiere O, Sleiman C, Menu Y, Fournier M. Clinical investigations immediate and long-term results of bronchial artery embolization for life-threatening hemoptysis. Chest 1999;115: 996-1001.

37. Eddy J B. Reviews clinical assessment and management of massive hemoptysis. Crit Care Med 2000;28:1642-7.

38. M L aughlin J L, H ankins J R. Current aspects of surgery for pulmonary tuberculosis. Ann Thorac Surg 1974;17:513-25. 